\title{
Apathy and depressive symptoms in older people and incident myocardial infarction, stroke, and mortality: a systematic review and meta-analysis of individual participant data
}

This article was published in the following Dove Press journal:

Clinical Epidemiology

\author{
Lisa SM Eurelings $1, *$ \\ Jan Willem van Dalen ${ }^{1, *}$ \\ Gerben ter Riet ${ }^{2}$ \\ Eric P Moll van Charante ${ }^{2}$ \\ Edo Richard ${ }^{1,3}$ \\ Willem A van Gool'

\section{On behalf of the ICARA Study Group} \\ 'Department of Neurology, \\ Academic Medical Center, University \\ of Amsterdam, Amsterdam, the \\ Netherlands; ${ }^{2}$ Department of General \\ Practice, Academic Medical Center, \\ University of Amsterdam, Amsterdam, \\ the Netherlands; ${ }^{3}$ Donders Institute \\ for Brain, Cognition and Behaviour, \\ Department of Neurology, Radboud \\ University Medical Centre, Nijmegen, \\ the Netherlands
}

*These authors contributed equally to this work

Correspondence: Jan Willem van Dalen Department of Neurology, Academic Medical Center, University of

Amsterdam, 9 Meibergdreef, Amsterdam II 00 DD, the Netherlands

Tel +3I 205666889

Fax +3I 205669290

Email j.vandalen@amc.nl
Background: Previous findings suggest that apathy symptoms independently of depressive symptoms measured using the Geriatric Depression Scale (GDS) are associated with cardiovascular disease (CVD) in older individuals.

Aims: To study whether apathy and depressive symptoms in older people are associated with future CVD, stroke, and mortality using individual patient-data meta-analysis.

Methods: Medline, Embase, and PsycInfo databases up to September 3, 2013, were systematically searched without language restrictions. We sought prospective studies with older (mean age $\geq 65$ years) community-dwelling populations in which the GDS was employed and subsequent stroke and/or CVD were recorded to provide individual participant data. Apathy symptoms were defined as the three apathy-related subitems of the GDS, with depressive symptoms the remaining items. We used myocardial infarction (MI), stroke, and all-cause mortality as main outcomes. Analyses were adjusted for age, sex, and MI/stroke history. An adaptation of the Newcastle-Ottawa scale was used to evaluate bias. Hazard ratios were calculated using onestage random-effect Cox regression models.

Results: Of the 52 eligible studies, 21 (40.4\%) were included, comprising 47,625 older people (mean age [standard deviation] 74 [7.4] years), over a median follow-up of 8.8 years. Participants with apathy symptoms had a $21 \%$ higher risk of MI (95\% confidence interval [CI] 1.08-1.36), a $37 \%$ higher risk of stroke (95\% CI 1.18-1.59), and a $47 \%$ higher risk of all-cause mortality (95\% CI 1.38-1.56). Participants with depressive symptoms had a comparably higher risk of stroke (HR 1.36, 95\% CI 1.18-1.56) and all-cause mortality (HR 1.44, 95\% CI 1.35-1.53), but not of MI (HR 1.08, 95\% CI 0.91-1.29). Associations for isolated apathy and isolated depressive symptoms were comparable. Sensitivity analyses according to risk of bias yielded similar results. Conclusion: Our findings stress the clinical importance of recognizing apathy independently of depressive symptoms, and could help physicians identify persons at increased risk of vascular disease.

Keywords: apathy, older people, depression, cardiovascular disease, meta-analysis, stroke, myocardial infarction

\section{Introduction}

A number of meta-analyses have reported that depression in older people is associated with an increased risk of cardiovascular disease (CVD). ${ }^{1-4}$ From a clinical point of view, it is important that specific symptom clusters within depression might be differentially related to incident CVD. ${ }^{5-7}$ One such symptom cluster is apathy, generally defined 
as disrupted motivation, resulting in reduced goal-driven behavior, cognition, and emotion, although exact definitions vary. We found that in community-dwelling older people, a cluster of three motivational symptoms related to apathy within the 15-item Geriatric Depression Scale (GDS15) ${ }^{8-10}$ was associated with an increased risk of incident CVD. There was no such association for the 12 remaining symptoms within the GDS15. ${ }^{11}$

This finding corroborated previously reported associations of apathy symptoms with a history of stroke, CVD, and concurrent cardiovascular (CV) risk factors. ${ }^{8,12}$ These findings are consistent with the "vascular apathy" hypothesis, which supposes vascular factors play an important role in the etiology of apathy in later life. ${ }^{12}$ Apathy symptoms can occur in both the context of depression and independently as an isolated syndrome of disturbed motivation, ${ }^{13}$ reportedly occurring in around $10 \%-20 \%$ of community-dwelling older people. ${ }^{8,14-16}$ However, apathy currently receives little prominence in diagnostic practice. Determining whether community-dwelling older people with apathy symptoms have an increased risk of $\mathrm{CV}$ events is important, since these individuals may be disinclined to engage actively in healthy behavior and be easily overlooked in general practice, because of a tendency to withdraw from medical care due to the nature of the condition.

To corroborate our findings regarding the relation between incident CVD and apathy symptoms, external validation is essential. For this purpose, the Initiative on Cardiovascular Disease Risk and Apathy (ICARA) was set up, which aimed to study the independent relationships of apathy and depressive symptoms with myocardial infarction (MI), stroke, and mortality risk, by pooling data from longitudinal cohort studies in community-dwelling older people worldwide. Given our previous findings and the vascular apathy hypothesis, we hypothesized that apathy and depressive symptoms would be independently and differentially associated with MI, stroke, and mortality risk.

\section{Methods}

We conducted a participant-data meta-analysis in accordance with the Preferred Reporting Items for Systematic Reviews and Meta-analyses of Individual Participant Data (PRISMA-IPD) guidelines and the recommendations made by Riley et al for conducting IPD analyses. ${ }^{17,18}$ The IPD protocol was registered in the PROSPERO database (http://www.crd.york.ac.uk/PROSPERO/display record. $\underline{\text { asp?ID }=\text { CRD42014013087\#.VWSK447tlBc, Supplementary }}$ materials). This study was judged exempt from review by the Amsterdam Academic Medical Center Medical Ethics Committee.

\section{Search strategy and selection criteria}

Using the Ovid platform, we searched the Medline, Embase, and PsycInfo databases up to and including September 3, 2013. We did not apply any language restrictions or restrictions on publication type or year. The full search strategy is described in the Supplementary material. In short, we used relevant keywords to search for longitudinal studies employing any version of the GDS and containing longitudinal data on CVD (including peripheral arterial disease [PAD], angina pectoris, and $\mathrm{MI}$ ) and/or stroke (including transient ischemic attack [TIA]). Furthermore, we screened the bibliographies of selected papers and contacted investigators of a potentially eligible study known through acquaintance.

We included published original research papers that studied samples of community-dwelling older people (mean age $\geq 65$ years) representative of the general population, employed a GDS version in which the three apathy items previously identified through several principal-component analyses were included, ${ }^{8,9}$ and investigated occurrence of stroke and/or CVD. There were no restrictions regarding duration of follow-up. Studies that included both communitydwelling and institutionalized participants were eligible only if they were able to distinguish between the two. We tried to maximize representation for the general population by excluding studies that selected participants based on frailty, disability, CV-risk profile, or any specific disease. We also excluded case-control studies, since the control groups of these studies are likely to form a particular selection, rather than a cohort representative of the general population. To balance the required demands of reference screening, study coordination, and data harmonization with the relevance of the data contribution we excluded dissertations, conference abstracts and studies with a sample size $\leq 100$.

Titles and abstracts of identified papers were screened independently by two reviewers (LE, JWD). Full texts of selected articles were retrieved and papers reporting on the same single study collated. Studies were assessed for eligibility independently by both reviewers. Discrepancies were resolved by consensus. A third reviewer was available for consultation where agreement could not be reached (ER). For studies that were cross-sectional but otherwise eligible, PubMed and the Internet were searched for any indication of longitudinal follow-up being available for the study population in question. If so, studies were considered eligible. 
Authors of eligible studies were invited to contribute data for our analyses. A reminder was sent after 1 month.

\section{Data, definitions, and statistics}

The variables requested and methods used to check data integrity are described in the Supplementary materials. Unless reported there, all statistical analyses were prespecified.

\section{Apathy and depressive symptoms}

We defined apathy symptoms as a score $\geq 2$ on the three apathy items of the GDS15 apathy subscale (GDS3A): "Have you dropped many of your activities and interests?", "Do you prefer to stay at home, rather than going out and doing new things?", and "Do you feel full of energy?" (reversecoded). ${ }^{8,9}$ These items have been reported to have a sensitivity of $69 \%$ and a specificity of $85 \%$ to detect apathy based on the customary cutoff $(>13)$ of the 14-item apathy scale in older adults. ${ }^{12}$ We defined depressive symptoms as a score $\geq 2$ on the 12 remaining items of the GDS15 (GDS12D). For studies employing GDS versions with $>15$ items, we selected the appropriate three and 12 items. We performed separate analyses for apathy symptoms, isolated apathy symptoms (concurrent GDS3A $\geq 2$ and GDS12D $\leq 1$ ), depressive symptoms, and isolated depressive symptoms (concurrent GDS12D $\geq 2$ and GDS3A $\leq 1$ ). These cutoffs were based on analyses in previous studies. ${ }^{11}$ We conducted sensitivity analyses in which the more specific $\geq 5$ cutoff for GDS12D depressive symptoms was used and isolated symptoms were defined as a score $\geq 2$ on one subscale and a score of 0 on the other. We excluded individuals with more than one missing item on the GDS3A and/or more than two missing items on the GDS12D from the analyses. Post hoc, a sensitivity analysis was performed with apathy and depressive scores as continuous predictors.

\section{Outcomes}

We used time to first fatal or nonfatal MI, first fatal or nonfatal stroke (TIA excluded), and mortality as primary outcomes. Regarding MI and stroke events, these could be both first ever (i.e. incident) or recurrent. Mixed-model Cox proportional-hazard regression was used to calculate hazard ratios (HRs). Mixed-model logistic regression analyses were used to repeat the primary analyses on MI and stroke with the inclusion of studies in which dates of events were unavailable. A sensitivity analysis was conducted, including TIA. Finally, in order to compare results with those of our previous studies, a composite CVD outcome comprising myocardial infarction (MI), angina pectoris (AP) and peripheral arterial disease (PAD) was analyzed. ${ }^{11}$ Because an exact date of onset may be difficult to establish in cases of AP and PAD, these analyses were performed using logistic regression models. Subanalyses were conducted for CV mortality (primary CV cause of death according to the ICD9 [390-459] or -10 [I00-I99]) and non-CV mortality (all other causes) separately. A detailed description of how vascular variables were harmonized is provided in the Supplementary material. To assess how HRs differed among studies, we plotted and pooled the separate results of fixed-effect Cox analyses in each study for all primary outcomes, using inverse variance-weighted DerSimonian and Laird random-effect models. To quantify heterogeneity, $I^{2}$ statistics were used.

\section{Confounders and interaction}

Three models were assessed for each outcome. Model 1 was unadjusted, model 2 adjusted for age and sex (in logistic regression additionally for study follow-up duration), and model 3 additionally adjusted for history of MI and/or stroke, because these may be confounders in the relationship of apathy and depressive symptoms with each outcome. ${ }^{11}$ Interaction analyses were performed to evaluate possible moderation by age, sex, and history of MI and/or stroke. To examine the effect of age on the observed relations in more detail, we performed subgroup analyses within tertiles based on age. Furthermore, subgroup analyses were performed in individuals without a history of MI and/or stroke to facilitate comparison with our previous analyses. ${ }^{11}$

\section{Statistical analysis}

Random-effect Cox proportional-hazard regression analyses were performed in $\mathrm{R}$ (version 3.2) using the Coxme package. ${ }^{19,20}$ Overall HRs for the IPD meta-analyses were calculated using a one-stage random-effect model, with independent random slopes in each study for each predictor to account for clustering of participants (ie, clusters based on study). ${ }^{21}$ For binary logistic regression analyses, similar models were used, employing the Lme4 package. ${ }^{22}$ The $\mathrm{R}$ codes used are described in more detail in the Supplementary material. Statistical analyses were two-tailed, and significance was set at $\alpha=0.05$. We plotted and pooled HRs within individual studies using the Meta package. ${ }^{23}$

\section{Bias assessment}

We assessed availability bias by comparing distributions of age, sex, and population sizes of participating and nonpartici- 
pating studies. Risk of bias was evaluated per study using an adapted version of the Newcastle-Ottawa quality-assessment scale for cohort studies, ${ }^{24}$ based on the following characteristics: method of outcome assessment for MI and stroke (selfreport only vs other methods); proportion with missing status with regard to occurrence of MI, stroke, and mortality (lower vs higher than the median proportion for that outcome); study design (observational vs experimental); and selected vs general populations according to independent evaluation by two reviewers (LE, JWD), with selection based on age $(\geq 80$ years only), sex, and/or recruitment strategy (specific sample types) (Table S1). Discrepancies were resolved by consensus and consultation with a third author (WAG) if necessary. Sensitivity analyses comparing studies with lower vs higher risk of bias were performed for each primary outcome for each criterion.

\section{Results}

Search results are depicted in Figure 1. A total of 21 (40.4\% of eligible) studies participated, ${ }^{25-45}$ comprising data from

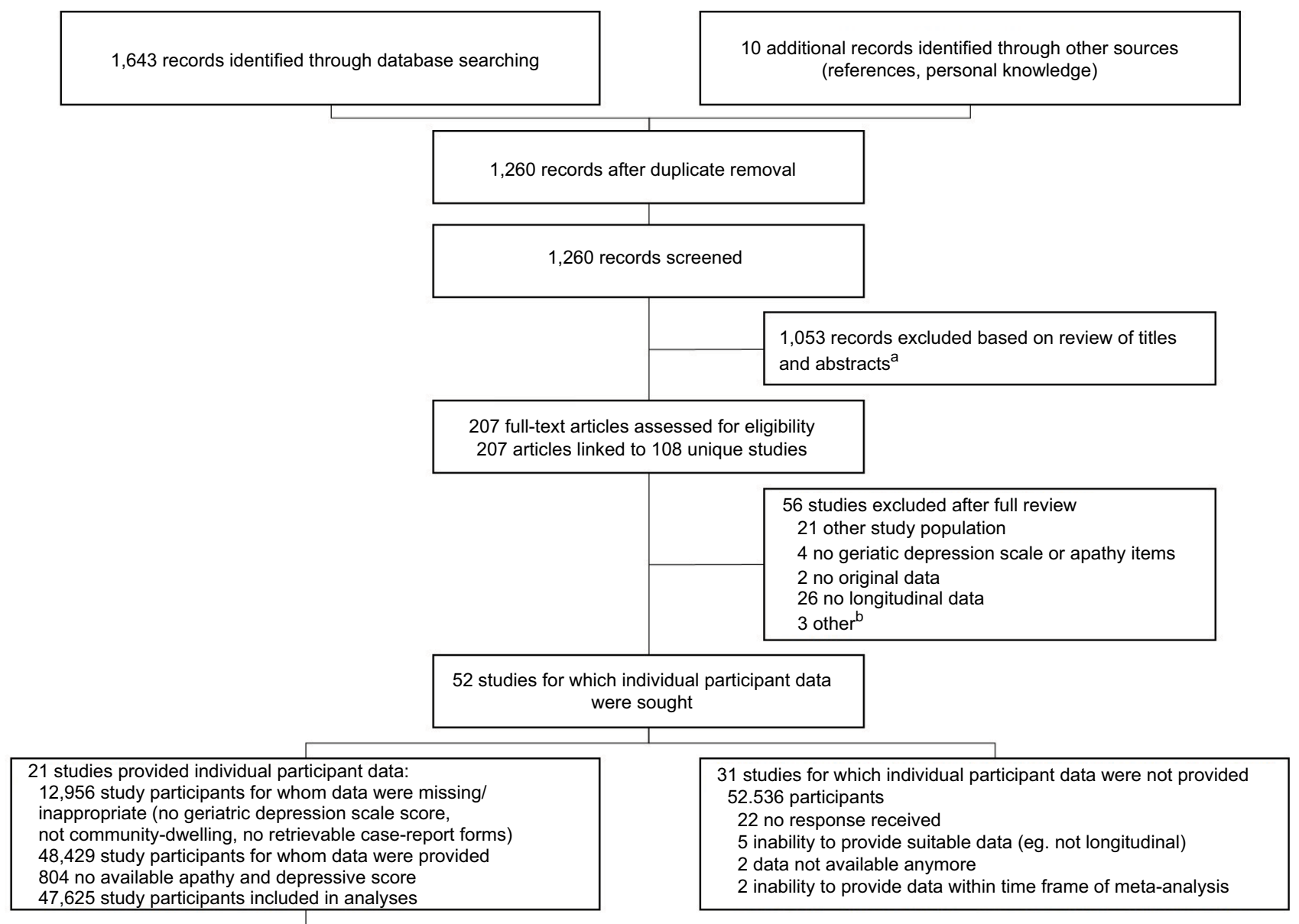

Time-to-event analysis on myocardial infarction

8 studies included in analysis

21,377 participants included in analysis

26,248 participants excluded from analysis (no [time-to-event] data on subsequent myocardial infarction)

Time-to-event analysis on stroke

10 studies included in analysis

23,337 participants included in analysis

24,288 participants excluded from analysis (no [time-to-event] data

on subsequent stroke)

Time-to-event analysis on mortality

21 studies included in analysis

46,859 participants included in analysis

766 participants excluded from analysis (no [time-to-event] data on mortality)

Figure I PRISMA diagram of study selection

Notes: ${ }^{a}$ Dissertation/conference abstract, $\leq 100$ study participants, no longitudinal data, case-control study, no original data, no geriatric depression scale or geriatric depression scale version without apathy items; 'one dissertation, one study with $\leq 100$ participants, and full text not available for one study. 
48,429 community-dwelling older people. One study used a slightly modified apathy item on the GDS15. Another study could only provide data on apathy, not on the depression items of the GDS15 (Supplementary material). After excluding participants with neither apathy nor depression scores $(n=804)$, the final sample consisted of 47,625 older (mean age $74 \pm 7.4$ [range 53-105] years) community-dwelling people (51.5\% women). The median follow-up for mortality was 8.8 years, yielding 360,306 person-years in total. In total, $31.1 \%(14,788$ of 47,566$)$ of participants had apathy symptoms, $32.4 \%(15,093$ of 46,624$)$ depressive symptoms, $13.8 \%(6,541$ of 47,243$)$ isolated apathy symptoms, $15.3 \%$ $(7,169$ of 46,947$)$ isolated depressive symptoms, and $16.7 \%$ $(7,911$ of 47,276$)$ both. Table 1 describes the general characteristics of the individual study and total ICARA samples. Table S1 describes details on study design, in/exclusion criteria, recruitment strategy, and MI and stroke assessment.

\section{Outcomes}

Time-to-event data were available for stroke in ten $(n=23,337)$ and for MI in eight $(\mathrm{n}=21,377)$ studies. MI occurred in $5.7 \%$ $(1,217)$ and stroke in $6.2 \%(1,452)$ of individuals. For participants with and without apathy symptoms, average 5-year risk was $27 \%$ vs $16.3 \%$ for mortality, $6 \%$ vs $3.9 \%$ for MI, and $7.4 \%$ vs $4.2 \%$ for stroke, respectively. Average 5-year risk comparing participants with and without depressive symptoms was $24.7 \%$ vs $17.3 \%$ for mortality, $4.5 \%$ vs $4.6 \%$ for MI, and $6.4 \%$ vs $4.8 \%$ for depressive symptoms, respectively. Data on fatal and nonfatal events were provided by all except two studies, which only supplied data on nonfatal events. ${ }^{27,45}$ No violations of the proportional-hazard assumption were identified through evaluation of survival curves and Schönfeld residuals. Adjusted for age, sex, and history of MI and/or stroke, presence of apathy symptoms increased the hazard of future MI by $21 \%$ (HR 1.21, 95\% confidence interval [CI] 1.08-1.36) and future stroke by 37\% (HR 1.37, 95\% CI 1.18-1.59) (Table 2). Depressive symptoms similarly increased the hazard of future stroke by $36 \%$ (HR 1.36 , 95\% CI 1.18-1.56), but increased the hazard of MI less substantially by $8 \%$, with CIs overlapping 0 (HR 1.08 , 95\% CI 0.91-1.29). Results for isolated apathy and isolated depressive symptoms were comparable to those for apathy and depressive symptoms, respectively.

Sensitivity analyses including TIA in analyses of stroke gave similar results (Table S2). Results of analyses using logistic regression instead of Cox regression models (allowing for four additional studies for MI and two for stroke) were also comparable (Table S3). The association between stroke and depressive symptoms was slightly attenuated when the GDS12D $\geq 5$ cutoff for depressive symptoms was applied. A stricter definition for isolated symptoms ( 0 points on the other subscale) slightly attenuated the association for isolated apathy symptoms with future MI and stroke (Tables S4 and S5). The hazard for the composite outcome of occurrence of MI, AP, and/or PAD was 33\% (95\% CI 1.20--1.49) higher for participants with apathy symptoms compared to those without and $8 \%$ for participants with depressive symptoms, with CIs overlapping 0 (95\% CI 0.85-1.37) (Table S6).

Time-to-event data with regard to all-cause mortality were provided by all studies ( $\mathrm{n}=46,859)$. During follow-up, 33.3\% $(15,626)$ of participants died. In the fully adjusted models, both apathy symptoms (HR 1.47, 95\% CI 1.38-1.56) and depressive symptoms (HR 1.44, 95\% CI 1.35-1.53) increased the hazard of mortality by nearly $50 \%$ (Table 2 ). Associations for isolated apathy and depressive symptom were similar.

Sensitivity analyses using the stricter isolated symptom definition and those using the GDS12D $\geq 5$ cutoff for depressive symptoms gave comparable results regarding mortality (Tables S7 and S8). ICD codes regarding cause of death were provided by 12 studies. HRs for apathy and depressive symptoms seemed slightly higher for non-CV (apathy HR 1.45 [95\% CI 1.34-1.57]; depressive HR 1.50 [95\% CI 1.35-1.66]) compared to CV mortality (apathy HR 1.38 [95\% CI 1.16-1.66]; depressive HR 1.33 [95\% CI 1.18-1.51]), and CIs of the estimates overlapped (Table S9).

When apathy symptoms were explored as categorical predictors, a dose-response relationship between increasing apathy scores and increasing HRs was found for all outcomes (Figure 2). Pooled analyses of HRs in individual studies gave similar results to the one-stage approach (summarized in Table 3, forest plots in Figures $\mathrm{S} 1-\underline{\mathrm{S} 3})$. Heterogeneity was low in analyses of MI (apathy $I^{2}=13 \%$, depressive $I^{2}=12 \%$, isolated apathy $I^{2}=37 \%$, isolated depressive $I^{2}=0$ ), low in analyses of stroke (all $I^{2}=0$ ), and moderate in analyses of mortality for overall symptoms (apathy $I^{2}=47 \%$, depressive $I^{2}=50 \%$ ), while low for isolated symptoms (both $I^{2}=0$ ). Funnel plots of these analyses showed no indication of publication bias (Figures S4- $\underline{\text { S6}}$ ).

\section{Interaction}

We observed interactions between age and (isolated) depressive symptoms (HR 0.98, 95\% CI 0.96-0.99; isolated symptoms, HR 0.97, 95\% CI 0.94-0.99) in their relationship with stroke. This suggests that the relation between (isolated) depressive symptoms and future stroke is stronger in younger participants. These interactions were consistently present 
Table I Characteristics of total ICARA population and individual study samples included in ICARA

\begin{tabular}{|c|c|c|c|c|c|}
\hline Study & Region & $\begin{array}{l}\text { GDS- } \\
\text { examination } \\
\text { date }\end{array}$ & Participants, $\mathbf{n}$ & $\begin{array}{l}\text { Age, mean } \\
\text { (SD), years }\end{array}$ & Women, n/total (\%) \\
\hline Total ICARA population & & $1990-2014$ & 47,625 & $74(7.4)$ & $24,544 / 47,625(51.5)$ \\
\hline Median (IQR) across studies & & & $\mathrm{I}, 735(958-3,303)$ & $74.4(72.2-78.3)$ & $56.6 \%(48.6 \%-61.2 \%)$ \\
\hline BELFRAIL study $(\mathrm{BFC} 80+)^{25}$ & Belgium & 2008-2009 & 499 & $84(3.5)$ & $302 / 499(60.5)$ \\
\hline Canadian Study on Health and Aging (CSHA $)^{26}$ & Canada & $1995-1997$ & $1,5 \mid 4$ & $82.5(6.7)$ & $874 / 1,514(57.7)$ \\
\hline Einstein Aging Study (EAS) ${ }^{27}$ & USA & $1998-2014$ & 1,924 & $78.2(5.4)$ & $1,189 / 1,924(61.8)$ \\
\hline Health in Men Study (HIMS) ${ }^{28}$ & Australia & $200 I-2004$ & 5,519 & $76.8(3.7)$ & $0 / 5,519(0)$ \\
\hline $\begin{array}{l}\text { Health, Well-Being, and Aging Study - Brazil } \\
\left(^{(S A B E)^{29}}\right.\end{array}$ & Brazil & 2000 & $\mathrm{I}, 863$ & $72.1(7.8)$ & $\mathrm{I}, 094 / \mathrm{I}, 863(58.7)$ \\
\hline Hertfordshire Cohort Study (HCS) ${ }^{30}$ & England & $1995-1996$ & 882 & $68.4(2.8)$ & $340 / 882(38.5)$ \\
\hline Hong Kong Old-Old Study (HKOOS) ${ }^{31}$ & China & $1991-1992$ & $\mathrm{I}, 540$ & $77.8(6.2)$ & $693 / 1,540(45)$ \\
\hline $\begin{array}{l}\text { Insufficienza Cardiaca negli Anziani Residenti a } \\
\text { Dicomano study (ICARe Dicomano) }\end{array}$ & Italy & $1995-1996$ & 634 & $73.7(6.4)$ & $374 / 634$ (59) \\
\hline $\begin{array}{l}\text { Intervention Project on Cerebrovascular } \\
\text { Diseases and Dementia in the Ebersberg } \\
\text { District (INVADE) }\end{array}$ & Germany & $200 I-2003$ & 3,837 & $67.1(7.7)$ & $2,256 / 3,857(58.8)$ \\
\hline $\begin{array}{l}\text { Invecchiamento Cerebrale in Abbiategrasso } \\
\text { study (InveCe.Ab) }{ }^{34}\end{array}$ & Italy & $2009-2011$ & 1,262 & $72.2(1.3)$ & $680 / 1,262(53.9)$ \\
\hline Italian Longitudinal Study on Aging (ILSA) ${ }^{35}$ & Italy & $1992-1993$ & 3,342 & $74.4(5.6)$ & I,578/3,342 (47.2) \\
\hline Leiden 85-Plus study $\left(\right.$ L85+) ${ }^{36}$ & the Netherlands & $1997-1999$ & 445 & $85(0)$ & $275 / 445(61.8)$ \\
\hline Mr Os and Ms Os study (MrMs Os) $)^{37}$ & China & $200 I-2003$ & 3,998 & $72.3(5.2)$ & $1,999 / 3,998(50)$ \\
\hline $\begin{array}{l}\text { Osservatorio Geriatrico Regione Campania } \\
\text { study }(\mathrm{OGC})^{38}\end{array}$ & Italy & $|990-199|$ & 1,250 & $74.1(6.3)$ & $707 / 1,250(56.6)$ \\
\hline $\begin{array}{l}\text { Outcomes of Sleep Disorders in Older Men } \\
\text { study (MrOS Sleep) })^{39}\end{array}$ & USA & $2003-2005$ & 3,130 & $76.4(5.6)$ & $0 / 3,130(0)$ \\
\hline Selenium and Cognitive Decline Study (SCDS) ${ }^{40}$ & China & $2006-2007$ & $\mathrm{I}, 735$ & $73.8(5.3)$ & $926 / 1,735(53.4)$ \\
\hline Singapore Longitudinal Ageing Study I (SLAS I) ${ }^{41}$ & Singapore & $2003-2005$ & 2,789 & $66.0(7.7)$ & $1,760 / 2,789(63.1)$ \\
\hline Singapore Longitudinal Ageing Study II (SLAS II) ${ }^{42}$ & Singapore & $2008-2013$ & 3,263 & $67.0(7.9)$ & $2,041 / 3,263(62.5)$ \\
\hline Study of Osteoporotic Fractures (SOF $)^{43}$ & USA & $1991-1994$ & 6,591 & $76.7(4.8)$ & $6,591 / 6,591(100.0)$ \\
\hline Sydney Memory and Ageing Study (MAS) ${ }^{44}$ & Australia & $2005-2007$ & 1,033 & $78.3(4.8)$ & $569 / 1,033(55.1)$ \\
\hline Treviso Longeva study (Trelong) ${ }^{45}$ & Italy & $2003-2004$ & 575 & $82.3(7.7)$ & $296 / 575(51.5)$ \\
\hline
\end{tabular}

Notes: aStudy samples included in the meta-analyses consisted of community-dwelling individuals with an available apathy and/or depression score. All samples populationbased. 'Numbers concern only studies included in time-to-event analysis. 'Study included in time-to-event analysis and logistic regression analysis. ${ }^{\circledR}$ Study included in logistic regression analysis only. elf a history of stroke excluding TIA was not available to create the composite variable history of Ml and/or stroke, a history of stroke including TIA was used instead if available. 'Due to time constraints in digitalizing individual GDS items from paper case-record forms, the ICARe Dicomano study provided data on the GDS3A items only within the time frame of this meta-analysis. 8Median of mortality follow-up calculated excluding deceased individuals. Number of participants with missing data within each study sample: age - CSHA 6; baseline apathy score - CSHA 2, HIMS 2, ILSA I3, INVADE 36, MAS 3, MrOS Sleep I, SABE-Brazil I, SLAS I I; baseline depression score - CSHA 4, EAS 2, HIMS 9, ICARe Dicomano634 (not available [NA]), ILSA 2I3, INVADE 40, MAS 4, SABE 86, SLAS I I, SOF 8; history of MI and/or stroke - BFC80+ 18, CSHA I,5I4 (NA), HCS 882 (NA), HKOOS I,540 (NA), ICARe Dicomano 634 (NA), ILSA 2, INVADE I9, InveCe.Ab 2, L85+ 5, MAS I3, OGC I,250 (NA), SABE-I,863 (NA), SCDS 2, SLASI 4, SLAS2 2, SOF 3, Trelong 575 (NA); subsequent MI - BFC80+ 65 (time to event [TTE] and logistic [L]), CSHA I,5I4 (NA), EAS 976 (TTE + L), HCS 882 (NA), HKOOS I,540 (NA), ICARe Dicomano 634 (NA), ILSA 218 (TTE + L), InveCe.Ab I88 (L), L85+ 2 (TTE + L), MAS I40 (L), MrMs Os I (TTE + L), MrOS Sleep 55 (TTE + L), OGC I,250 (NA), SABE I,863 (NA), SCDS I,735 (NA), SLASI 2,789 (NA), SLAS2 3,263 (NA), SOF 404 (L), Trelong 275 (L); subsequent stroke - BFC80+ 54 (TTE + L), CSHA I,5 I4 (NA), EAS 964 (TTE+L), HCS 882 (NA), HIMS 18 (TTE + L), HKOOS I,540 (NA), ICARe Dicomano 634 (NA), ILSA 216 (TTE $+\mathrm{L}$ ), InveCe.Ab 203 (TTE + L), MAS I 40 (TTE + L), MrMs Os I (TTE + L), MrOS Sleep 56 (TTE + L), OGC I,250 (NA), SCDS I,735 (NA), SABE 843 (L), SLASI 2,789 (NA), SLAS2 3,263 (NA), SOF 6,59I (NA), Trelong 275 (L); number deceased - CSHA I,57, EAS 4, HCS 8, HKOOS 83, ILSA 217, MrOS Sleep I0I, SABE I73, SCDS23.

Abbreviations: GDS, Geriatric Depression Scale; MI, myocardial infarction; TIA, transient ischemic attack; ICARA, Initiative on Cardiovascular Disease Risk and Apathy.

across studies $\left(I^{2}=0\right)$. Interaction effects between age and both apathy and depressive symptoms (both HRs $0.99,95 \%$ CI 0.98-0.99) were present for mortality, suggesting the relationships were stronger in younger individuals. These interactions were also consistent across studies (apathy symptoms, $I^{2}=32.4 \%$; depressive symptoms, $I^{2}=22.9 \%$ ). Inclusion of these interaction terms into the fully adjusted models did not meaningfully alter any of the conclusions (data not shown). In subgroup analyses within tertiles based on age $(<72,72-77,>77$ years), the relationship between apathy and both MI and stroke was stronger in older subgroups. The relationship between depressive symptoms and stroke appeared stronger in younger subgroups (Tables S10-S12) Subanalyses in participants without a history of MI and/or stroke gave results similar to the main analyses (Tables S13 and $\underline{\mathrm{S} 14}$ ). 


\begin{tabular}{|c|c|c|c|c|c|c|}
\hline $\begin{array}{l}\text { Baseline apathy } \\
\text { symptoms } \\
\text { (GDS3A } \geq 2 \text { ), } \\
\text { n/total (\%) }\end{array}$ & $\begin{array}{l}\text { Baseline depressive } \\
\text { symptoms } \\
\text { (GDSI 2D } \geq 2 \text { ), } \\
\text { n/total (\%) }\end{array}$ & $\begin{array}{l}\text { History of MI } \\
\text { and/or stroke, } \\
\text { n/total (\%) }\end{array}$ & $\begin{array}{l}\text { Subsequent } \\
\text { MI, n/total (\%) }\end{array}$ & $\begin{array}{l}\text { Subsequent } \\
\text { stroke, } \\
\text { n/total (\%) }\end{array}$ & $\begin{array}{l}\text { Deceased, } \\
\text { n/total (\%) }\end{array}$ & $\begin{array}{l}\text { Follow-up } \\
\text { mortality, } \\
\text { median (IQR), } \\
\text { years }^{\text {g }}\end{array}$ \\
\hline $14,788 / 47,566(3 \mid . I)$ & $15,093 / 46,624(32.4)$ & $4,987 / 39,297(12.7)$ & $\mathrm{I}, 2 \mathrm{I} 7 / 2 \mathrm{I}, 377(5.7)^{\mathrm{b}}$ & $\mathrm{I}, 452 / 23,337(6.2)^{\mathrm{b}}$ & $15,626 / 46,859(33.3)$ & $8.8(5.3-10.9)$ \\
\hline $31.8 \%(21.4 \%-42.2 \%)$ & $31.3 \%(21.1 \%-47.3 \%)$ & $13.6 \%(7.7 \%-18.2 \%)$ & $3.3 \%(2.3 \%-9.1 \%)^{b}$ & $5 \%(3.4 \%-7.3 \%)^{\mathrm{b}}$ & $38.1 \%(22.8 \%-48.6 \%)$ & $8.5(5-11.2)$ \\
\hline 205/499 (4I.I) & I86/499 (37.3) & $89 / 48 \mid(18.5)$ & $12 / 434(2.8)^{c}$ & $25 / 445(5.6)^{c}$ & $201 / 499(40.3)$ & $5.1(5-5.3)$ \\
\hline $540 / 1,512(35.7)$ & $486 / 1,510(32.2)$ & - & - & - & $529 / 1,357$ (39) & $5(4.7-5.2)$ \\
\hline $727 / 1,924(37.8)$ & $562 / 1,922(29.2)$ & $348 / 1,924$ (18.1) & $22 / 948(2.3)^{c}$ & $37 / 960(3.9)^{c}$ & $732 / 1,920(38.1)$ & $8(0-16)$ \\
\hline $2,372 / 5,517(43)$ & $1,223 / 5,510(22.2)$ & $\mathrm{I}, 010 / 5,519(18.3)^{\mathrm{e}}$ & $623 / 5,519(11.3)^{c}$ & $600 / 5,501(10.9)^{c}$ & $\mathrm{I}, 653 / 5,5 \mathrm{I} 9(30)$ & $7.4(6.7-8.1)$ \\
\hline 379/1,862 (20.4) & $824 / 1,777$ (46.4) & - & - & $138 / I, 020(13.5)^{d}$ & $814 / 1,690(48.2)$ & $10.7(10.4-10.9)$ \\
\hline 175/882 (19.8) & I54/882 (I7.5) & - & - & - & $350 / 874(40)$ & I5.I (14.7-15.4) \\
\hline$I, 050 / I, 540(68.2)$ & I,2 I0/I,540 (78.6) & - & - & - & $7 \mid 2 / 1,457$ (48.9) & $4.7(3-10.2)$ \\
\hline $189 / 634(29.8)$ & $-{ }^{f}$ & - & - & - & I77/634 (27.9) & $8.5(8.3-8.5)$ \\
\hline I,57|/3,80I (4I.3) & $880 / 3,797(23.2)$ & $276 / 3,8 \mid 8(7.2)$ & $89 / 3,837(2.3)^{c}$ & $202 / 3,837(5.3)^{c}$ & $876 / 3,837(22.8)$ & $11.2(10.1-11.6)$ \\
\hline $140 / 1,262$ (II.I) & $4 \mid 7 / 1,262(33)$ & $171 / 1,260(13.6)^{\mathrm{e}}$ & $17 / 1,074(1.6)^{d}$ & $14 / 1,059(1.3)^{c}$ & $45 / 1,262(3.6)$ & $2.2(2.1-2.3)$ \\
\hline I,059/3,329 (3I.8) & $1,767 / 3,129(56.5)$ & $405 / 3,340(12.1)$ & $118 / 3,124(3.8)^{c}$ & $147 / 3,126(4.7)^{c}$ & $\mathrm{I}, 258 / 3,125(40.3)$ & $8.1(3.6-8.5)$ \\
\hline I I 3/445 (25.4) & I I0/445 (24.7) & $69 / 440$ (15.7) & $43 / 443(9.7)^{c}$ & $45 / 445(10.1)^{c}$ & $424 / 445(95.3)$ & $13.5(12.9-13.9)$ \\
\hline $895 / 3,998$ (22.4) & I,893/3,998 (47.3) & $545 / 3,998(13.6)^{\mathrm{e}}$ & $81 / 3,997(2)^{c}$ & $173 / 3,997(4.3)^{c}$ & $970 / 3,998(24.3)$ & II.3 (|0.7-|| I.8) \\
\hline $682 / 1,250(54.6)$ & $883 / 1,250(70.6)$ & - & - & - & $658 / 1,250(52.6)$ & $12.2(12.2-12.2)$ \\
\hline $928 / 3,129(29.7)$ & $503 / 3,130(16.1)$ & $625 / 3,130(20)$ & $229 / 3,075(7.4)^{c}$ & $194 / 3,074(6.3)^{c}$ & I,055/3,029 (34.8) & $9.5(9.3-9.8)$ \\
\hline $901 / 1,735$ (51.9) & $988 / 1,735(56.9)$ & $90 / 1,733(5.2)$ & - & - & $304 / 1,712(17.8)$ & $4.5(4.5-4.5)$ \\
\hline $239 / 2,788(8.6)$ & $844 / 2,788(30.3)$ & $188 / 2,785(6.8)^{e}$ & - & - & $327 / 2,789$ (1I.7) & $9.9(9-10.6)$ \\
\hline $70 / 3,263(2.1)$ & $365 / 3,263$ (11.2) & $253 / 3,261(7.8)^{e}$ & - & - & $142 / 3,263(4.4)$ & $3.6(2.5-5)$ \\
\hline$|, 8| 1 / 6,59 \mid(27.5)$ & $1364 / 6,583(20.7)$ & $767 / 6,588(11.6)^{\mathrm{e}}$ & $463 / 6,187(7.5)^{d}$ & - & $3,797 / 6,591$ (57.6) & $15.7(13.5-16.5)$ \\
\hline $504 / 1,030(48.9)$ & $162 / 1,029(15.7)$ & $15|/|, 020(14.8)$ & $27 / 893(3)^{d}$ & $15 / 893(1.7)^{c}$ & $235 / 1,033(22.7)$ & $7(5.8-7.1)$ \\
\hline 238/575 (4I.I) & $272 / 575(47.3)$ & - & $1 \mathrm{I} / 300(3.7)^{\mathrm{d}}$ & $16 / 300(5.3)^{d}$ & $367 / 575(63.8)$ & $10.4(10.1-10.8)$ \\
\hline
\end{tabular}

\section{Risk of bias}

Overall, studies that did not participate in ICARA $(\mathrm{n}=31)$ were smaller than those included ( $\mathrm{n}=21)$, had slightly older study populations, and included relatively more women (Supplementary material). Sensitivity analyses comparing studies with higher versus lower risk of bias generally did not give any substantial deviations from the main analysis results, with association magnitudes being similar (Tables S15- $\underline{\text { S21 }}$ ). However, the evidence was poor in some categorizations, due to substantially smaller sample sizes (eg, experimental study designs [Table S15], self-reported outcomes [Table S19], and high percentage of missing outcomes [Table S20]). Sensitivity analyses with apathy and depressive-symptom scores as continuous predictors (Table S22) showed similar results: in models adjusted for age, sex, and history of MI and/or stroke, the hazard for participants with apathy symptoms for future MI increased, with an 11\% per point increase (HR 1.11, 95\%
CI 1.04-1.17), for stroke with a $15 \%$ per point increase (HR $1.15,95 \%$ CI $1.05-1.26$ ), and for mortality with a $24 \%$ per point increase (HR 1.24, 95\% CI 1.20-1.28). Associations were comparable for isolated apathy symptoms. Depressive symptoms gave a 5\% increase per point for future stroke (HR $1.05,95 \%$ CI 1.00-1.10) and $9 \%$ for mortality (HR 1.09, 95\% CI 1.07-1.11). The hazard of MI increased less substantially, by $3 \%$ per point increase, with CIs overlapping 0 (HR 1.03, 95\% CI 0.99-1.08).

\section{Availability of data}

The data used in this paper are available from each individual participating study. However, restrictions apply to the availability of these data, which are not publicly available and were used under license for the current study. Data are however available from the authors upon reasonable request and with the permission of each individual study included. 
Table 2 Associations of apathy and depressive symptoms with subsequent myocardial infarction, stroke, and all-cause mortality

\begin{tabular}{|c|c|c|c|c|c|c|}
\hline & \multicolumn{6}{|c|}{ Myocardial infarction* } \\
\hline & \multicolumn{2}{|l|}{ Model I } & \multicolumn{2}{|l|}{ Model $2^{f}$} & \multicolumn{2}{|l|}{ Model $3^{g}$} \\
\hline & Events/total, $\mathbf{n}$ & HR (95\% Cl) & Events/total, $n$ & HR (95\% Cl) & Events/total, $n$ & HR (95\% CI) \\
\hline Apathy & $1,213 / 21,326$ & $1.41(1.25-1.58)$ & $1,213 / 21,326$ & $1.29(1.15-1.45)$ & $1,211 / 21,290$ & $1.21(1.08-1.36)$ \\
\hline Apathy, isolated ${ }^{b}$ & $861 / 14,467$ & $1.46(1.26-1.69)$ & $861 / 14,467$ & $1.31(1 . \mid 3-1.51)$ & $859 / 14,446$ & $1.24(1.08-1.44)$ \\
\hline Depression ${ }^{c}$ & $1,204 / 21,129$ & $1.14(0.94-1.37)$ & $1,204 / 21,129$ & I.II (0.92-I.34) & $\mathrm{I}, 202 / 2 \mathrm{I}, 094$ & $1.08(0.91-1.29)$ \\
\hline \multirow[t]{4}{*}{ Depression, isolated $^{d}$} & $682 / 13,870$ & $1.17(0.95-1.44)$ & $682 / 13,870$ & $1.17(0.95-1.44)$ & $681 / 13,852$ & $1.14(0.93-1.40)$ \\
\hline & \multicolumn{6}{|l|}{ Stroke** } \\
\hline & \multicolumn{2}{|l|}{ Model I } & \multicolumn{2}{|l|}{ Model $2^{f}$} & \multicolumn{2}{|l|}{ Model $3^{g}$} \\
\hline & Events/total, $n$ & HR (95\% Cl) & Events/total, $n$ & HR (95\% Cl) & Events/total, $n$ & HR (95\% Cl) \\
\hline Apathy ${ }^{a}$ & $1,449 / 23,284$ & $1.62(1.40-1.87)$ & $1,449 / 23,284$ & $1.43(1.23-1.67)$ & $1,449 / 23,232$ & $1.37(1.18-1.59)$ \\
\hline Apathy, isolated ${ }^{b}$ & $928 / 15,928$ & $1.57(1.37-1.80)$ & $928 / 15,928$ & $1.38(1.2-1.58)$ & $928 / 15,892$ & $1.33(1.15-1.52)$ \\
\hline Depression ${ }^{c}$ & $1,430 / 23,086$ & $1.46(1.26-1.70)$ & $1,430 / 23,086$ & $1.39(1.20-1.61)$ & $1,430 / 23,035$ & $1.36(1.18-1.56)$ \\
\hline \multirow[t]{4}{*}{ Depression, isolated $^{d}$} & $762 / 15,291$ & $1.25(1.04-1.50)$ & $762 / 15,291$ & $1.22(1.01-1.46)$ & $762 / 15,263$ & I. $18(0.99-1.42)$ \\
\hline & \multicolumn{6}{|c|}{ All-cause mortality**** } \\
\hline & \multicolumn{2}{|l|}{ Model I } & \multicolumn{2}{|l|}{ Model $2^{f}$} & \multicolumn{2}{|l|}{ Model 38 } \\
\hline & Events/total, $n$ & HR (95\% Cl) & Events/total, $n$ & HR (95\% Cl) & Events/total, $n$ & HR (95\% Cl) \\
\hline Apathy ${ }^{a}$ & $|5,6| 5 / 46,802$ & $1.75(I .63-1.87)$ & $15,6 \mid 2 / 46,796$ & $\mathrm{I} .52(\mathrm{I} .44-\mathrm{I} .6 \mathrm{I})$ & | I,984/38,898 & $\mathrm{I} .47(\mathrm{I} .38-\mathrm{I} .56)$ \\
\hline Apathy, isolated ${ }^{b}$ & $9,198 / 31,119$ & $1.73(1.65-1.82)$ & $9,198 / 31,118$ & $1.46(1.40-1.53)$ & $7,733 / 27,576$ & $1.44(1.36-1.51)$ \\
\hline Depression ${ }^{c}$ & $15,268 / 45,877$ & $1.55(1.43-1.67)$ & $|5,265 / 45,87|$ & $1.46(1.37-1.56)$ & ||$, 86|/ 38,69|$ & $1.44(1.35-1.53)$ \\
\hline Depression, isolated $^{d}$ & $8,774 / 31,647$ & $1.38(1.27-1.50)$ & $8,77 \mid / 31,642$ & $1.33(1.25-1.42)$ & $7,042 / 27,380$ & $1.36(1.28-1.44)$ \\
\hline
\end{tabular}

Notes: aScore $\geq 2$ on the three-item apathy subscale of the Geriatric Depression Scale (GDS3A) and any depression score; ${ }^{\text {bscore }} \geq 2$ on the GDS3A in participants with one or no depressive symptoms; 'score $\geq 2$ on the 12-item depression subscale of the GDS (GDSI2D) and any apathy score; 'score $\geq 2$ on the GDSI2D in participants with one or no apathy symptoms; ' analysis: *models I-3 - BFC80+, EAS, HIMS, ILSA, INVADE, L85+, MrMs Os, MrOS Sleep; **models I-3 - BFC80+, EAS, HIMS, ILSA, INVADE, InveCe.Ab, L85+, MAS, MrMs Os, MrOS Sleep; ***models I and 2 - BFC80+, CSHA, EAS, HCS, HIMS, HKOOS, ICARe Dicomano (apathy analysis only because of unavailable depression scores), ILSA, INVADE, InveCe.Ab, L85+, MAS, MrMs Os, MrOS Sleep, OGC, SABE, SLASI, SLAS2, SCDS, SOF, Trelong; model 3 - BFC80+, EAS, HIMS, ILSA, INVADE, InveCe.Ab, L85+, MAS, MrMs Os, MrOs Sleep, SCDS, SLASI, SLAS2, SOF (unavailable history of myocardial infarction and/or stroke for the other studies).

Abbreviations: $\mathrm{Cl}$, confidence interval; $\mathrm{HR}$, hazard ratio.

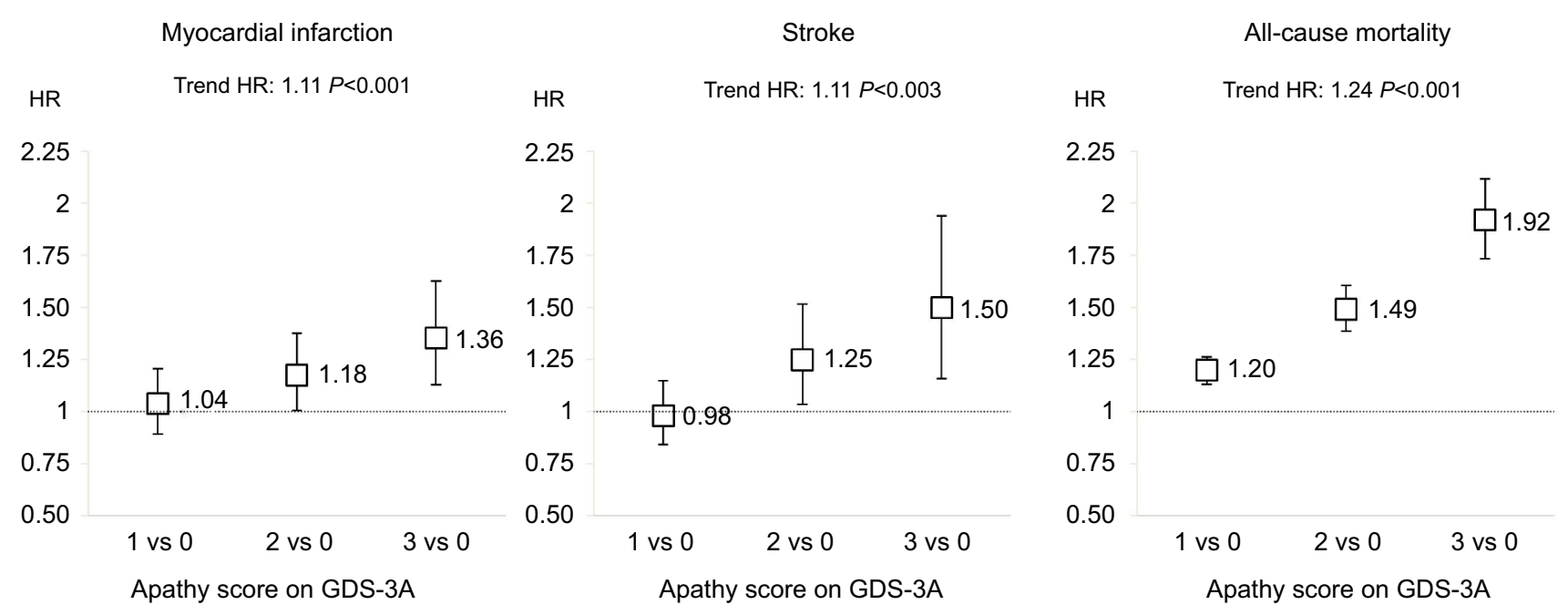

Figure 2 HRs for myocardial infarction, stroke, and all-cause mortality by apathy score in individuals without depressive symptoms.

Notes: Results from Cox proportional-hazard analyses with GDS3A score as predictor adjusted for baseline age, sex, and history of myocardial infarction and/or stroke in individuals without depressive symptoms (GDSI2 score $\leq 1$ ).

Abbreviation: GDS, Geriatric Depression Scale. 
Table 3 Two-stage analyses of associations of apathy and depressive symptoms with subsequent myocardial infarction, stroke, and all-cause mortality

\begin{tabular}{|c|c|c|c|c|c|c|}
\hline & \multicolumn{6}{|c|}{ Myocardial infarction* } \\
\hline & \multicolumn{2}{|l|}{ Model I } & \multicolumn{2}{|l|}{ Model $2^{f}$} & \multicolumn{2}{|l|}{ Model 3g } \\
\hline & HR (95\% Cl) & $I^{2}$ & HR (95\% Cl) & $I^{2}$ & HR (95\% Cl) & $I^{2}$ \\
\hline Apathy & $1.40(1.24-1.59)$ & 5 & $1.28(1.10-1.49)$ & 18 & $1.21(1.05-1.40)$ & 13 \\
\hline Apathy, isolated & $1.52(1.26-1.84)$ & 19 & $1.41(1.13-1.76)$ & 33 & $1.35(\mid .07-1.7 I)$ & 37 \\
\hline Depressionc & $1.16(0.96-1.39)$ & 28 & I.I $15(0.97-1.36)$ & 21 & $1.12(0.95-1.31)$ & 12 \\
\hline \multirow[t]{4}{*}{ Depression, isolated } & $1.21(0.99-1.49)$ & 0 & $1.21(0.98-1.49)$ & 0 & $1.18(0.96-1.46)$ & 0 \\
\hline & \multicolumn{6}{|l|}{ Stroke** } \\
\hline & \multicolumn{2}{|l|}{ Model I } & \multicolumn{2}{|l|}{ Model $2^{f}$} & \multicolumn{2}{|l|}{ Model 38 } \\
\hline & HR (95\% Cl) & $I^{2}$ & HR (95\% Cl) & $I^{2}$ & HR (95\% Cl) & $I^{2}$ \\
\hline Apathy ${ }^{a}$ & $1.71(1.54-1.91)$ & 0 & $1.49(1.32-1.69)$ & 10 & $1.45(1.3-1.61)$ & 0 \\
\hline Apathy, isolated & $1.59(1.39-1.83)$ & 0 & $1.39(1.21-1.60)$ & 0 & $1.34(1.17-1.54)$ & 0 \\
\hline Depressionc & $1.55(1.29-1.75)$ & 26 & $1.44(1.25-1.66)$ & 17 & $1.43(1.27-1.60)$ & 0 \\
\hline \multirow[t]{4}{*}{ Depression, isolated } & $1.3(1.08-1.56)$ & I & $1.27(1.05-1.52)$ & 0 & $1.24(1.03-1.49)$ & 0 \\
\hline & \multicolumn{6}{|c|}{ All-cause mortality**** } \\
\hline & \multicolumn{2}{|l|}{ Model I } & \multicolumn{2}{|l|}{ Model $2^{f}$} & \multicolumn{2}{|l|}{ Model 3g } \\
\hline & HR (95\% CI) & $P^{2}$ & HR (95\% Cl) & $I^{2}$ & HR (95\% Cl) & $I^{2}$ \\
\hline Apathy ${ }^{a}$ & $1.73(1.62-1.85)$ & 68 & $1.52(1.44-1.60)$ & 48 & $1.46(1.38-1.56)$ & 47 \\
\hline Apathy, isolated & $1.72(1.63-1.81)$ & 9 & $1.46(1.39-1.53)$ & 0 & $1.44(|.36-| .5 \mid)$ & 0 \\
\hline Depression $^{c}$ & $1.54(1.43-1.67)$ & 77 & $1.46(1.37-1.56)$ & 67 & $1.44(1.35-1.53)$ & 50 \\
\hline Depression, isolated ${ }^{d}$ & $1.39(1.27-1.52)$ & 58 & $1.33(1.25-1.43)$ & 32 & $1.38(1.3-1.46)$ & 0 \\
\hline
\end{tabular}

Notes: Results from two-stage analyses by pooling results of Cox proportional-hazard analyses within individual studies using DerSimonian and Laird random-effect models (forest plots in Figures SI-S3). ${ }^{\text {SScore }} \geq 2$ on the three-item apathy subscale of the Geriatric Depression Scale (GDS3A) and any depression score; 'score $\geq 2$ on the GDS3A in participants with one or no depressive symptoms; 'score $\geq 2$ on the 12 -item depression subscale of the GDS (GDSI2D) and any apathy score; ${ }^{d}$ score $\geq 2$ on the GDSI2D in participants with one or no apathy symptoms; 'unadjusted; fadjusted for baseline age and sex; gadjusted for baseline age, sex, and history of myocardial infarction and/ or stroke. Included in analysis: *models I-3 - BFC80+, EAS, HIMS, ILSA, INVADE, L85+, MrMs Os, MrOS Sleep; **models I-3 - BFC80+, EAS, HIMS, ILSA, INVADE, InveCe.Ab, L85+, MAS, MrMs Os, MrOS Sleep; ***models I and 2 - BFC80+, CSHA, EAS, HCS, HIMS, HKOOS, ICARe Dicomano (apathy analysis only because of unavailable depression scores), ILSA, INVADE, InveCe.Ab, L85+, MAS, MrMs Os, MrOS Sleep, OGC, SABE-Brazil, SLASI, SLAS2, SCDS, SOF, Trelong; model 3 - BFC80+, EAS, HIMS, ILSA, INVADE, InveCe.Ab, L85+, MAS, MrMs Os, MrOs Sleep, SCDS, SLASI, SLAS2, SOF (unavailable history of myocardial infarction and/or stroke for the other studies). Abbreviation: GDS, Geriatric Depression Scale

\section{Discussion}

In this IPD meta-analysis of a large sample of communitydwelling older people, we found that apathy symptoms, irrespectively of concurrent depressive symptoms, were associated with a higher risk of MI, stroke, and all-cause CV and non-CV mortality. Depressive symptoms were related to a similarly increased risk of stroke and mortality outcomes, but not of MI. These observed associations were independent of age, sex, and history of MI/stroke.

The current study has several strengths. Large-scale meta-analysis of IP data is considered the "gold standard" for meta-analyses, ${ }^{18}$ reducing the potential for ecological bias compared to meta-analyses synthesizing individual study results. ${ }^{46} \mathrm{An}$ assortment of studies were included with regard to country of origin and demographic characteristics, increasing the generalizability of our findings. The risk of publication bias seems limited, given that none of the included studies originally aimed to assess the association between apathy symptoms and vascular outcomes or mortality. Nevertheless, publication bias of the relationship between depression and
CVD could have indirectly influenced our results. Funnel plots of within-study associations, however, suggested the risk of publication bias playing a role in our findings is low. One-stage analyses were performed, which are recommended above two-stage methods, since their statistical approach is more exact, have more power for detection of interaction, and allow for better control of confounding and accounting for heterogeneity by allowing controlling for covariates at both the participant and study level. ${ }^{21,46}$ Finally, the data were extensively explored in a range of sensitivity and interaction analyses.

Although it may be considered a limitation that the apathy and depression subscales have not been validated against a clinical diagnosis, ${ }^{10}$ the intention of our study was not to formally diagnose apathy and/or depression, but rather to explore whether degrees of apathy and depressive symptoms were differentially associated with future vascular disease and mortality. It is important to note that our results pertain to the symptoms in the GDS15, and may not be generalizable to all depressive and apathy symptoms in a clinical interview. 
We could not adjust for all potential confounders, including (chronic) vascular comorbidity, functional and cognitive impairment, socioeconomic status, and education, potentially leading to residual confounding. However, adjustment for some of these characteristics could have led to overadjustment, since they could also function as mediators. We tried to reduce the risk that individuals with severe cognitive and functional impairment were included in our analyses by excluding institutionalized individuals. In addition, several studies have found associations of apathy in older people with adverse outcomes independently of disability and cognition. ${ }^{11,16,47}$

Although we were able to acquire IPD for a large number of older people, more than half the eligible studies did not participate. These studies seemed to have slightly different population characteristics, suggesting that not including their data may have had some impact on our results. Nonparticipating studies were smaller, had more women, and slightly older populations. Since older age was a mediator of the associations with apathy symptoms, inclusion of these older cohorts could have decreased the magnitude of the unadjusted associations. Furthermore, although we incorporated studies from all over the world, more variety in population provenance may have allowed investigation into how geography affects these relations, since it has been shown that the exact construct of apathy symptoms within the GDS may differ among cultures. ${ }^{48}$ Finally, since we did not have longitudinal data regarding the development of apathy and depressive symptoms over time, we cannot ascertain whether their stability has an influence on the risk of subsequent unfavorable clinical outcomes.

That apathy symptoms in this IPD meta-analysis were associated with subsequent MI (and also PA and PAD) independently of depressive symptoms corroborates our previous report of a specific association for apathy symptoms with an increased risk of CVD ${ }^{11}$ and concurs with the vascular apathy hypothesis. ${ }^{12}$ This IPD meta-analysis is (to our knowledge) the first to study the association between apathy symptoms and subsequent MI systematically. Previous studies assessing the reverse association consistently reported that a history of vascular disease predicted greater apathy, but not depression scores. ${ }^{12,49}$ That apathy symptoms are specifically associated with future MI is in line with findings in other studies that suggest that motivation-related somatic rather than moodrelated depressive symptoms are related to adverse CV outcomes in older individuals. ${ }^{6,50,51}$ We found no association between depressive symptoms and future MI, which may be contrary to results of several traditional meta-analyses. ${ }^{1-4}$ However, since no discrimination was made between apathy and depressive symptomatology in these studies, it is possible that those associations were at least partly driven by apathy.

Both apathy and depressive symptoms were associated with a an approximately 37\%-higher risk of future stroke. This is contrary to our previous single-cohort study, in which no associations between either apathy or depressive symptoms and higher future stroke risk were found. ${ }^{11}$ This discrepancy can most likely be explained by the far greater statistical power of the current analysis. In addition, the present study population was slightly younger on average, and both our own results and the previous literature suggest that the relationship between depressive symptoms and future stroke is stronger in younger people. ${ }^{52,53}$ The association of depressive symptoms with future stroke is in line with several meta-analyses that - although not distinguishing between apathy and depressive symptoms - have shown that depression and somewhat less so depressive symptoms are independent risk factors for stroke, increasing the risk by $20 \%-45 \% .^{52-55}$

We found an approximately 45\% higher hazard for both apathy and depressive symptoms with all-cause mortality. A similarly increased risk of mortality in people with depression has been demonstrated by several studies. ${ }^{56-59}$ Studies that have examined the relation between apathy symptoms and mortality in general community-dwelling older populations are sparse, employed different methods of apathy assessment, and mostly studied specific populations, eg, those with frailty, heart disease, or dementia. ${ }^{60-64}$

The mechanisms by which apathy and depressive symptoms are related to future adverse vascular outcomes are unknown. Conceivably, both causal and noncausal effects play a role. Deleterious health behaviors and CV-risk factors may be induced by apathy and depression and thus partly mediate the reported associations with unfavorable outcomes. ${ }^{65-70}$ Other mediators may also be of influence, eg, reduced compliance with $\mathrm{CV}$-risk management. ${ }^{70}$ Alternatively, the observed associations may be due to a common etiology underlying vascular disease, apathy, and depressive symptoms. It is imaginable that apathy and/or depressive symptoms are markers of underlying subclinical (cerebral) atherosclerosis, which is a strong risk factor for vascular events. ${ }^{70}$ Previous studies have associated apathy and depressive symptoms with the presence of white-matter lesions in older individuals, also without clinical depression. ${ }^{71-75}$ Although our previous research suggests that the relation- 
ship between apathy symptoms and future vascular events is independent of CV-risk factors, ${ }^{11,67}$ conceivably apathy symptoms reflect cerebrovascular damage by long-standing elevated CV risk, eg, midlife hypertension. If apathy is indeed a marker of cerebrovascular damage, such risk factors could be considered confounders underlying the relation between apathy and incident disease. Furthermore, although apathy predicts future vascular events independently of vascular event history, vascular events are a risk factor for apathy. This suggests that the temporal relationship between apathy and vascular events could be bidirectional, corresponding with previous findings that apathy symptoms can also be preceded by CVD. ${ }^{12}$

Our finding that symptoms of apathy gave an approximately $20 \%$ increased hazard of MI, while depressive symptoms did not seem clearly to increase this hazard, may have several explanations. Apathy symptoms may be more specific markers of underlying cerebrovascular disease, as suggested by the stronger relation with incident stroke, and thus more strongly associated with other incident vascular disease. Alternatively, apathy symptoms may be more sensitive to prodromal $\mathrm{CV}$ disease, since individuals with poor $\mathrm{CV}$ condition may tend to be less outgoing and feel less energetic. The relationship among apathy and depressive symptoms and mortality may be mediated by the increased occurrence of major vascular events, including MI and stroke, which are among the most important causes of death worldwide. ${ }^{76}$ Alternatively, both types of symptoms could be manifestations of other underlying conditions that give a higher risk of mortality.

The dose-response relationship between apathy symptoms and subsequent stroke, MI and mortality, independent of depressive symptoms, together with previous analyses suggesting that apathy predicts CVD over and above the presence of $\mathrm{CV}$-risk factors ${ }^{67}$ stress that recognition of apathy symptoms in older people is important, regardless of depressive symptoms. Although apathy symptoms may manifest themselves in the context of depression, our research adds to the expanding body of evidence highlighting apathy as an important standalone syndrome. ${ }^{8,12,49,67,77-81}$ Distinct associations were found between apathy symptoms and a number of patient-important CV outcomes, including MI, AP, and PAD. Participants with two or more apathy symptoms had a $20 \%-30 \%$-increased risk of these outcomes, independently of concurrent depressive symptoms. Individuals with a maximum apathy score ( $10.6 \%$ of our study population) had an $88 \%$ increased hazard of mortality. These findings imply that more attention on recognizing, detecting, and managing apathy in general practice is warranted. This is especially relevant, since patients with apathy are less likely to engage actively in CV-risk prevention and health care. ${ }^{82}$

It is also important to recognize apathy as an entity distinct from depression, since treatment with selective serotonin-reuptake inhibitors (SSRIs) has not only been found to be ineffective but could also potentially aggravate apathy. ${ }^{83-85}$ Given the common and potentially serious side effects of SSRI treatment in older people, this is especially undesirable. ${ }^{86,87}$ If treatment of symptoms could mitigate the increased risks, based on the risks in the symptom-free populations and fully adjusted models, successful apathy treatment could decrease the absolute 5-year risks for these individuals from $4.7 \%$ to $3.9 \%$ for $\mathrm{MI}$ and from $5.8 \%$ to $4.2 \%$ for stroke. Depressive-symptom treatment would decrease these risks by $5.0 \%$ to $4.6 \%$ for MI and from $6.5 \%$ to $4.8 \%$ for stroke. Increased awareness of apathy in research settings is instrumental in elucidating its specific etiological role in $\mathrm{CV}$ disease and increasing the knowledge of its clinical correlates.

In conclusion, this IPD meta-analysis provides substantial evidence that apathy symptoms in community-dwelling older people are associated with an increased risk of future stroke, MI, and mortality, independently of concurrent depressive symptoms. It suggests depressive symptoms are associated with a similarly increased risk of stroke and mortality, but not substantially of MI. These independent associations between apathy symptoms and patient-important outcomes stress the necessity of recognizing apathy in older people independently of depressive symptoms. Future studies may investigate whether these associations are specific for apathy symptoms according to the GDS or also apply similarly to apathy and depressive symptoms according to other scales. Future research could evaluate the effects of change in the presence of these symptoms over time, aim at unraveling the mechanisms underlying their associations with adverse outcomes in later life, and examine whether interventions can prevent or reduce their occurrence and clinical consequences.

\section{ICARA study-group authors and collaborators}

The ICARA study-group authors are as follows: Osvaldo P Almeida, MD, PhD, Tiago S Alexandre, PhD, Bernhard T Baune, MD, PhD, Horst Bickel, PhD, Francesco Cacciatore, MD, PhD, Cyrus Cooper, FMedSci, Ton (A)JM de Craen, $\mathrm{PhD}^{\dagger}$, Jean-Marie Degryse, $\mathrm{MD}, \mathrm{PhD}$, Mauro Di Bari, MD, PhD, Yeda A Duarte, PhD, Liang Feng, PhD, Nicola Ferrara, MD, Leon Flicker, MB, BS, PhD, FRACP, Maurizio Gallucci, 
MD, Antonio Guaita, MD, Stephanie L Harrison, MPH, Mindy J Katz, MPH, Maria L Lebrão, PhD†, Jason Leung, MSc, Richard B Lipton, MD, Marta Mengoni, MD, PhD, Tze Pin Ng, MD, PhD, Truls Østbye, MD, PhD, Francesco Panza, MD, PhD, Letizia Polito, PhD, Dirk Sander, MD, PhD, Vincenzo Solfrizzi, MD, PhD, Holly E Syddall, PhD, Roos $\mathrm{C}$ van der Mast, $\mathrm{MD}, \mathrm{PhD}$, Bert Vaes, MD, PhD, Jean Woo, MD, Kristine Yaffe, MD. 'Ton de Craen and Maria Lebrão passed away during the drafting of the manuscript.

The ICARA study-group collaborators are as follows: Sujuan Gao, PhD; Suzanne C. Ho, PhD; Joan Lindsay, PhD; Aprille Sham, MSc; Simone Reppermund, PhD; Frederick W. Unverzagt, $\mathrm{PhD}$. We gratefully acknowledge these individuals for their contribution to the acquisition of the study data and critical consideration of the manuscript. No compensation was received by any of these individuals for contribution to this study.

\section{ICARA study-group author and collaborator affiliations}

Department of Neurology, Academic Medical Center, University of Amsterdam, Amsterdam, TheNetherlands (Eurelings, van Dalen, Richard, van Gool), Department of General Practice, Academic Medical Center, University of Amsterdam, Amsterdam, Netherlands (ter Riet, Moll van Charante), Department of Neurology, Radboud University Medical Centre, Nijmegen, the Netherlands (Richard), Golgi Cenci Foundation, Abbiategrasso, Italy (Guaita, Polito), Center for Aging Research and Education, Duke NUS Medical School, Singapore (Østbye), Centre for Healthy Brain Ageing, Faculty of Medicine, School of Psychiatry, University of New South Wales, Sydney, Australia (Reppermund), Centre Medical Research, Western Australian Centre for Health \& Ageing, University of Western Australia, Perth, Australia (Flicker), Cognitive Impairment Center, Health District of Treviso, Local Health Authority 9 of Treviso, Treviso, Italy (Gallucci), Department of Basic Medicine, Neuroscience, and Sense Organs, Neurodegenerative Disease Unit, Pia Fondazione Cardinale G Panico, University of Bari Aldo Moro, Tricase, Italy (Panza), Department of Biostatistics, Indiana University School of Medicine, Indiana University, Indianapolis, IN, USA (Gao), Department of Developmental Disability Neuropsychiatry, Faculty of Medicine, School of Psychiatry, University of New South Wales, Sydney, Australia (Reppermund), Department of Epidemiology and Biostatistics, California Pacific Medical Center Research Institute, University of California, San Francisco, CA, USA (Harrison, Yaffe), Department of Epidemiology and
Population Health, Albert Einstein College of Medicine, New York, NY, USA (Lipton), Department of Epidemiology, Faculty of Public Health, University of São Paulo, São Paulo, Brazil (Lebrão), Department of Experimental and Clinical Medicine, Research Unit of Medicine of Aging, University of Florence, Florence, Italy (Di Bari, Mengoni), Department of Geriatric Medicine, Royal Perth Hospital, Perth, Australia (Flicker), Department of Geriatrics, Azienda OspedalieroUniversitaria Careggi, Florence, Italy (Di Bari), Department of Gerontology and Geriatrics, Leiden University Medical Center, Leiden, the Netherlands (De Craen), Department of Gerontology, Center for Biological and Health Sciences, Federal University of São Carlos, São Carlos, Brazil (Alexandre), Department of Health Sciences and System Research, Duke NUS Medical School, National University of Singapore, Singapore (Feng), Department of Medical-Surgical Nursing, University of São Paulo, São Paulo, Brazil (Duarte), Department of Medicine and Therapeutics, Prince of Wales Hospital, The Chinese University of Hong Kong, Hong Kong (Woo), Department of Neurology, Albert Einstein College of Medicine, New York, NY, USA (Lipton, Katz), Department of Neurology, Benedictus Krankenhaus Tutzing, Technische Universität München, Tutzing, Germany (Sander), Department of Psychiatry and Behavioral Science, Albert Einstein College of Medicine, New York, NY, USA (Lipton), Department of Psychiatry and Clinical Neurosciences, Royal Perth Hospital, University of Western Australia, Perth, Australia (Almeida), Department of Psychiatry and Psychotherapy, Klinikum Rechts der Isar, Technical University of Munich, Munich, Germany (Bickel), Department of Psychiatry, Collaborative Antwerp Psychiatric Research Institute (CAPRI), University of Antwerp, Antwerp, Belgium (van der Mast), Department of Psychiatry, Indiana University School of Medicine, Indianapolis, IN, USA (Unverzagt), Department of Psychiatry, Leiden University Medical Center, Leiden, Netherlands (van der Mast), Department of Psychological Medicine, Yong Loo Lin School of Medicine, National University of Singapore, Singapore, (Feng, Ng), Department of Public Health and Primary Care, Katholieke Universiteit Leuven, Leuven, Belgium (Degryse, Vaes), Department of Translational Medical Sciences, University of Naples "Federico II", Naples, Italy (Cacciatore, Ferrara), Departments of Psychiatry and Neurology, University of California, San Francisco, CA, USA (Yaffe), Discipline of Psychiatry, Royal Adelaide Hospital, University of Adelaide, Adelaide, Australia (Baune), Duke Global Health Institute, Duke University, Durham, NC, USA (Østbye), Harry Perkins Institute for Medical Research, Western Australian Centre 
for Health \& Ageing, Royal Perth Hospital, University of Western Australia, Perth, Australia (Almeida), Institut de Recherche Santé et Société, Université Catholique de Louvain, Brussels, Belgium (Degryse, Vaes), Interdisciplinary Department of Medicine, Geriatric Medicine and Memory Unit, Azienda Ospedaliero-Universitaria Consorziale Policlinico di Bari, University of Bari Aldo Moro, Bari, Italy (Solfrizzi), Interdisciplinary Geriatric Research Foundation, Treviso, Italy (Gallucci), Jockey Club School of Public Health and Primary Care, The Chinese University of Hong Kong, Hong Kong, (Ho, Leung, Sham), Medical Research Council Lifecourse Epidemiology Unit, University of Southampton, Southampton, UK (Cooper, Syddall), National Institute for Health Research Southampton Biomedical Research Centre, University of Southampton and University Hospital Southampton NHS Foundation Trust, Southampton, UK (Cooper), National Institute for Health Research Musculoskeletal Biomedical Research Unit, University of Oxford, Oxford, UK (Cooper), Salvatore Maugeri Foundation, Scientific Institute of Telese Terme, Telese Terme, Italy (Cacciatore, Ferrara), School of Epidemiology, Public Health and Preventive Medicine, University of Ottawa, Ottawa, Canada (Lindsay), School of Medicine and Pharmacology, University of Western Australia, Perth, Australia (Flicker).

\section{ICARA participating studies}

Australia - Health in Men Study, Sydney Memory and Ageing Study; Belgium - BELFRAIL Study; Brazil - Health, Well-Being, and Aging Study; Canada - Canadian Study on Health and Aging; China - Hong Kong Old-Old Study, Mr Os and Ms Os Study, Selenium and Cognitive Decline Study; Germany - Intervention Project on Cerebrovascular Diseases and Dementia in the Ebersberg District; Italy Insufficienza Cardiaca negli Anziani Residenti a Dicomano Study, Invecchiamento Cerebrale in Abbiategrasso Study, Italian Longitudinal Study on Aging, Osservatorio Geriatrico Regione Campania Study, Treviso Longeva (Trelong) Study; Singapore - Singapore Longitudinal Ageing Study I, Singapore Longitudinal Ageing Study II; the Netherlands Leiden 85-Plus Study; UK - Hertfordshire Cohort Study; USA - Einstein Aging Study; Outcomes of Sleep Disorders in Older Men Study; Study of Osteoporotic Fractures.

\section{Acknowledgments}

The Sydney Memory and Ageing Study was supported by the National Health and Medical Research Council (NHMRC; grants 350833, 510175, and 510124) and the Australian Research Council (DP0774213, DP0773584, and LP0669645). The Health, Well-Being, and Aging Study - Brazil was supported by the Foundation of Research Support of São Paulo (FAPESP; grants 1999/05125-7, 2005/54947-2, and 2009/53778-3). The Intervention Project on Cerebrovascular Diseases and Dementia in the Ebersberg District was supported by the Allgemeine Ortskrankenkasse Bayern, the German Stroke Foundation, Bayer Vital GmbH, Berlin-Chemie AG, Organon Pharmaceuticals, Ratiopharm $\mathrm{GmbH}$, Sanofi Synthelabo GmbH, and Teva Pharmaceutical Industries Ltd. The Mr Os and Ms Os Study was supported by The Hong Kong Jockey Club Charities Trust and The SH Ho Centre for Gerontology and Geriatrics. The Singapore Longitudinal Ageing Studies were sponsored by the Biomedical Research Council (grants 03/1/21/17/214 and 08/1/21/19/567). The Hertfordshire Cohort Study was sponsored by the Medical Research Council UK. The Hong Kong Old-Old Study was sponsored by the Croucher Foundation and Hong Kong Health Services Research (grant 411009). The Health in Men Study was sponsored by the National Health and Medical Research Council (grants 279408, 379600, 403963, 513823, 540403, 540504, 540405, 634492, 1021416, 1045710, and 1060557). The Canadian Study of Health and Aging was initially funded through the Canadian federal government's Seniors' Independence Research Program (1998). The program funds were administered by the National Health Research and Development Program of Health Canada. Supplementary funding was administered by the Medical Research Council of Canada. After 1998, funding for the core study was obtained from the Canadian Institutes for Health Research (grant MOP-42530) and supplementary funding for the caregiver component was provided under grant MOP-43945. Throughout the study, additional funding was obtained for supplementary components and for personnel funding from provincial governments, research granting agencies, and the private sector. Funding from the pharmaceutical industry contributed to the collection and analysis of biological samples, to the caregiver substudy, to personnel support via training awards, and access to provincial health care plan data. Support was also provided by the Pfizer Corporation Inc, Bayer Canada Inc, Janssen-Ortho Inc, and Merck Frosst Canada \& Co. The study was coordinated through the University of Ottawa, which provided administrative support and facilities for the coordinating center, and the Division of Aging and Seniors, Health Canada. The Insufficienza Cardiaca negli Anziani Residenti a Dicomano study was funded by Regione Toscana. The Selenium and Cognitive Decline Study was supported by the National Institutes of Health/National Institute on Aging (grant R01 
AG019181). The BELFRAIL study (B40320084685) is funded by an unconditional grant from Fondation Louvain. Fondation Louvain is the support-group unit of the Université Catholique de Louvain, which is in charge of developing education and research projects for the university by collecting gifts from corporations, foundations, and alumni. The Treviso Longeva study was supported by Regione del Veneto (grant DGR 13/12/2002, 3604, project number 76), Fondazione Cassamarca, Fondazione Veneto Banca, the Province of Treviso, and the Municipality of Treviso. The Study of Osteoporotic Fractures (SOF) is supported by National Institutes of Health funding. The National Institute on Aging (NIA) provides support under grant numbers R01 AG005407, R01 AR35582, R01 AR35583, R01 AR35584, R01 AG005394, R01 AG027574, and R01 AG027576. The Osteoporotic Fractures in Men (MrOS) study is supported by National Institutes of Health funding. The following institutes provide support: the National Institute on Aging (NIA), the National Institute of Arthritis and Musculoskeletal and Skin Diseases (NIAMS), the National Center for Advancing Translational Sciences (NCATS), and NIH Roadmap for Medical Research under the grant numbers U01 AG027810, U01 AG042124, U01 AG042139, U01 AG042140, U01 AG042143, U01 AG042145, U01 AG042168, U01 AR066160, and UL1 TR000128. The National Heart, Lung, and Blood Institute (NHLBI) provides funding for the MrOS Sleep ancillary study Outcomes of Sleep Disorders in Older Men under the grant numbers R01 HL071194, R01 HL070848, R01 HL070847, R01 HL070842, R01 HL070841, R01 HL070837, R01 HL070838, and R01 HL070839. The Einstein Aging Study is supported by the National Institute on Aging (NIA) grant P01 AGO3949. The De Leiden 85-Plus study was supported by several unrestricted grants from the Netherlands Organisation of Scientific Research (ZonMw) and the Ministry of Health, Welfare, and Sports. Dr Eurelings received an Academic Medical Center PhD Scholarship (2011/1190). The funders of the study had no role in study design, data collection, data analysis, data interpretation, or writing of the report.

We gratefully acknowledge Francesca Mazzella, MD, PhD (Department of Translational Medical Sciences, Federico II University of Naples and Emergency Department, Antonio Cardarelli Hospital, Naples, Italy) for completing the mortality follow-up for the Osservatorio Geriatrico Regione Campania (OGC) study, Michele Cellurale, MD (Department of Translational Medical Sciences, Federico II University of Naples, Naples, Italy) for database management of the OGC study, Pasquale Abete, MD, PhD (Department of Translational Medical Sciences, Federico II University of Naples, Naples, Italy) for his contribution to study design and coordination of the OGC study, Graeme J Hankey, MD, FRACP (School of Medicine and Pharmacology, University of Western Australia, Perth, WA, Australia), Bu B Yeap, MBBS, PhD (School of Medicine and Pharmacology, University of Western Australia, Perth, Australia), Jonathan Golledge, MD, MChir, FRACS, FRCS (Queensland Research Centre for Peripheral Vascular Disease, College of Medicine and Dentistry, James Cook University, Cairns, QLD, Australia) for their contribution to obtain funding and data collection for the Health in Men study; all investigators for the first and second phase of the Canadian Study on Health and Aging (CSHA; listed in http://www.ncbi.nlm.nih.gov/ pmc/articles/PMC1486712/pdf/cmaj00286-0095.pdf and http://www.neurology.org/content/55/1/66.full.pdf) for their contribution to study design, data collection, and coordination of the CSHA. The National Registry of Diseases Office of the Health Promotion Board of Singapore is gratefully acknowledged for providing mortality data for the Singapore Longitudinal Ageing Studies. No compensation was received by any individual for contribution to this study.

\section{Author contributions}

All authors read and approved the final manuscript and were responsible for the study concept and design, acquisition/analysis/interpretation of data, critical revision of the manuscript for important intellectual content, administrative/ technical/material support, and agreed to be accountable for all aspects of the work. LSME and JWD were responsible for drafting the manuscript and statistical analysis, and EPMC, ER, and WAG for study supervision. JWD had full access to all the data in the study and had final responsibility for the decision to submit for publication.

\section{Disclosure}

The authors report no conflicts of interest in this work.

\section{References}

1. van der Kooy K, van Hout H, Marwijk H, Marten H, Stehouwer C, Beekman A. Depression and the risk for cardiovascular diseases: systematic review and meta analysis. Int J Geriatr Psychiatry. 2007;22(7): 613-626.

2. Rugulies R. Depression as a predictor for coronary heart disease: a review and meta-analysis. Am J Prev Med. 2002;23(1):51-61.

3. Nicholson A, Kuper H, Hemingway H. Depression as an aetiologic and prognostic factor in coronary heart disease: a meta-analysis of 6362 events among 146538 participants in 54 observational studies. Eur Heart J. 2006;27(23):2763-2774.

4. Gan Y, Gong Y, Tong X, et al. Depression and the risk of coronary heart disease: a meta-analysis of prospective cohort studies. $B M C$ Psychiatry. 2014;14:371. 
5. Baune BT, Stuart M, Gilmour A, et al. The relationship between subtypes of depression and cardiovascular disease: a systematic review of biological models. Transl Psychiatry. 2012;2:e92.

6. Hawkins MA, Callahan CM, Stump TE, Stewart JC. Depressive symptom clusters as predictors of incident coronary artery disease: a 15-year prospective study. Psychosom Med. 2014;76(1):38-43.

7. Freak-Poli R, Mirza SS, Franco OH, Ikram MA, Hofman A, Tiemeier H. Positive affect is not associated with incidence of cardiovascular disease: a population-based study of older persons. Prev Med. 2015;74:14-20.

8. Ligthart SA, Richard E, Fransen NL, et al. Association of vascular factors with apathy in community-dwelling elderly individuals. Arch Gen Psychiatry. 2012;69(6):636-642.

9. Mitchell J, Mathews HF, Yesavage JA. A multidimensional examination of depression among the elderly. Res Aging. 1993;15(2):198-219.

10. Bertens AS, Moonen JE, de Waal MW, et al. Validity of the three apathy items of the Geriatric Depression Scale (GDS3A) in measuring apathy in older persons. Int J Geriatr Psychiatry. 2017;32(4):421-428.

11. Eurelings LS, Ligthart SA, van Dalen JW, Moll van Charante EP, van Gool WA, Richard E. Apathy is an independent risk factor for incident cardiovascular disease in the older individual: a population-based cohort study. Int J Geriatr Psychiatry. 2014;29(5):454-463.

12. van der Mast RC, Vinkers DJ, Stek ML, et al. Vascular disease and apathy in old age: the Leiden 85-Plus study. Int $J$ Geriatr Psychiatry. 2008;23(3):266-271.

13. Robert P, Onyike CU, Leentjens AF, et al. Proposed diagnostic criteria for apathy in Alzheimer's disease and other neuropsychiatric disorders. Eur Psychiatry. 2009;24(2):98-104.

14. Levy ML, Cummings JL, Fairbanks LA, et al. Apathy is not depression. J Neuropsychiatry Clin Neurosci. 1998;10(3):314-319.

15. Landes AM, Sperry SD, Strauss ME. Prevalence of apathy, dysphoria, and depression in relation to dementia severity in Alzheimer's disease. J Neuropsychiatry Clin Neurosci. 2005;17(3):342-349.

16. Ayers E, Shapiro M, Holtzer R, Barzilai N, Milman S, Verghese J. Symptoms of apathy independently predict incident frailty and disability in community-dwelling older adults. J Clin Psychiatry. 2017;78(5):e529-e536.

17. Stewart LA, Clarke M, Rovers M, et al. Preferred reporting items for systematic review and meta-analyses of individual participant data: the PRISMA-IPD. JAMA. 2015;313(16):1657-1665.

18. Riley RD, Lambert PC, Abo-Zaid G. Meta-analysis of individual participant data: rationale, conduct, and reporting. BMJ. 2010;340:c221.

19. R Project. R: a language and environment for statistical computing Available from: http://www.R-project.org. Accessed February 4, 2018

20. Therneau TM 2015. Coxme: Mixed Effects Cox Models. R package version 2.2-5. Available from: http://CRAN.R-project.org/package=coxme. Accessed June 15, 2015.

21. Debray TP, Moons KG, Abo-Zaid GM, Koffijberg H, Riley RD. Individual participant data meta-analysis for a binary outcome: one-stage or two-stage? PloS One. 2013;8(4):e60650.

22. Bates D, Maechler M, Bolker B, et al. lme4: linear mixed-effects models using Eigen and S4. 2015. Available from: http://cran.r-project.org/ package $=1 m e 4$. Accessed February 4, 2018.

23. Schwarzer G. Meta: general package for meta-analysis. Available from: http://cran.r-project.org/package=meta. Accessed February 4, 2018

24. Wells GA, Shea B, O'Connell D, et al. The Newcastle-Ottawa Scale (NOS) for assessing the quality of nonrandomised studies in metaanalyses. Available from: http://www.ohri.ca/programs/clinical_epidemiology/oxford.asp. Accessed February 4, 2018.

25. Vaes B, Pasquet A, Wallemacq P, et al. The BELFRAIL (BFC80+) study: a population-based prospective cohort study of the very elderly in Belgium. BMC Geriatr. 2010;10:39.

26. McDowell I, Hill G, Lindsay J. An overview of the Canadian Study of Health and Aging. Int Psychogeriatr. 2001;13 Supp 1:7-18

27. Sanders AE, Wang C, Katz M, et al. Association of a functional polymorphism in the cholesteryl ester transfer protein (CETP) gene with memory decline and incidence of dementia. JAMA. 2010;303(2):150-158.
28. Norman PE, Flicker L, Almeida OP, Hankey GJ, Hyde Z, Jamrozik K. Cohort profile: the Health in Men Study (HIMS). Int J Epidemiol. 2009;38(1):48-52.

29. Alexandre TS, Corona LP, Nunes DP, Santos JL, Duarte YA, Lebrão ML. Gender differences in incidence and determinants of disability in activities of daily living among elderly individuals: SABE study. Arch Gerontol Geriatr. 2012;55(2):431-437.

30. Thompson C, Syddall H, Rodin I, Osmond C, Barker DJ. Birth weight and the risk of depressive disorder in late life. Br J Psychiatry. $2001 ; 179: 450-455$.

31. Ho SC, Woo J, Chan SS, Yuen YK, Sham A. Risk factors for falls in the Chinese elderly population. J Gerontol A Biol Sci Med Sci. 1996;51(5):M195-M198.

32. Di Bari M, Marchionni N, Ferrucci L, et al. Heart failure in community-dwelling older persons: aims, design and adherence rate of the ICARe Dicomano project - an epidemiologic study. J Am Geriatr Soc. 1999;47(6):664-671.

33. Scherpinski U, Bickel H, Gnahn H, Förstl H, Conrad B, Sander D. [Intervention project on cerebrovascular diseases and dementia in the Ebersberg district (INVADE): rationale and design]. Nervenarzt. 2002;73(12):1199-1204. German.

34. Guaita A, Colombo M, Vaccaro R, et al. Brain aging and dementia during the transition from late adulthood to old age: design and methodology of the "Invece.Ab" population-based study. BMC Geriatr. 2013;13:98.

35. Maggi S, Zucchetto M, Grigoletto F, et al. The Italian Longitudinal Study on Aging (ILSA): design and methods. Aging. 1994;6(6):464-473.

36. der Wiel AB, van Exel E, de Craen AJ, et al. A high response is not essential to prevent selection bias. results from the Leiden 85-plus study. J Clin Epidemiol. 2002;55(11):1119-1125.

37. Wong SY, Kwok T, Woo J, et al. Bone mineral density and the risk of peripheral arterial disease in men and women: results from $\mathrm{Mr}$ and $\mathrm{Ms}$ Os, Hong Kong. Osteoporos Int. 2005;16(12):1933-1938.

38. Cacciatore F, Gallo C, Ferrara N, et al. Morbidity patterns in aged population in southern Italy: a survey sampling. Arch Gerontol Geriatr. 1998;26(3):201-213.

39. Blackwell T, Yaffe K, Ancoli-Israel S, et al. Association of sleep characteristics and cognition in older community-dwelling men: the MrOS sleep study. Sleep. 2011;34(10):1347-1356

40. Gao S, Jin Y, Hall KS, et al. Selenium level and cognitive function in rural elderly Chinese. Am J Epidemiol. 2007;165(8):955-965.

41. Niti M, Yap KB, Kua EH, Tan CH, Ng TP. Physical, social and productive leisure activities, cognitive decline and interaction with APOE- $\varepsilon 4$ genotype in Chinese older adults. Int Psychogeriatr. 2008;20(2):237-251.

42. Feng L, Chong MS, Lim WS, et al. Metabolic syndrome and amnestic mild cognitive impairment: Singapore Longitudinal Ageing Study-2 findings. J Alzheimers Dis. 2013;34(3):649-657.

43. Cummings SR, Nevitt MC, Browner WS, et al. Risk factors for hip fracture in white women. N Engl J Med. 1995;332(12):767-773.

44. Sachdev PS, Brodaty H, Reppermund S, et al. The Sydney Memory and Ageing Study (MAS): methodology and baseline medical and neuropsychiatric characteristics of an elderly epidemiological nondemented cohort of Australians aged 70-90 years. Int Psychogeriatr. 2010;22(8):1248-1264.

45. Gallucci M, Ongaro F, Bresolin F, et al. The Treviso Longeva (Trelong) study: a biomedical, demographic, economic and social investigation on people 70 years and over in a typical town of north-east of Italy. Arch Gerontol Geriatr. 2007;44 Suppl 1:173-192.

46. Thomas D, Radji S, Benedetti A. Systematic review of methods for individual patient data meta-analysis with binary outcomes. BMC Med Res Methodol. 2014;14:79.

47. Geda YE, Roberts RO, Mielke MM, et al. Baseline neuropsychiatric symptoms and the risk of incident mild cognitive impairment: a population-based study. Am J Psychiatry. 2014;171(5):572-581.

48. Kim G, DeCoster J, Huang CH, Bryant AN. A meta-analysis of the factor structure of the Geriatric Depression Scale (GDS): the effects of language. Int Psychogeriatr. 2013;25(1):71-81. 
49. Sugawara N, Yasui-Furukori N, Umeda T, et al. Ankle brachial pressure index as a marker of apathy in a community-dwelling population. Int $J$ Geriatr Psychiatry. 2011;26(4):409-414.

50. Carroll AJ, Carnethon MR, Liu K, et al. Interaction between smoking and depressive symptoms with subclinical heart disease in the Coronary Artery Risk Development in Young Adults (CARDIA) study. Health Psychol. 2017;36(2):101-111.

51. Azevedo RM, Roest AM, Hoen PW, de Jonge P. Cognitive/affective and somatic/affective symptoms of depression in patients with heart disease and their association with cardiovascular prognosis: a meta-analysis. Psychol Med. 2014;44(13):2689-2703.

52. Salaycik KJ, Kelly-Hayes M, Beiser A, et al. Depressive symptoms and risk of stroke: the Framingham Study. Stroke. 2007;38(1):16-21.

53. Pan A, Sun Q, Okereke OI, Rexrode KM, Hu FB. Depression and risk of stroke morbidity and mortality: a meta-analysis and systematic review. JAMA. 2011;306(11):1241-1249.

54. Dong JY, Zhang YH, Tong J, Qin LQ. Depression and risk of stroke: a meta-analysis of prospective studies. Stroke. 2012;43(1):32-37.

55. Li M, Zhang XW, Hou WS, Tang ZY. Impact of depression on incident stroke: a meta-analysis. Int J Cardiol. 2015;180:103-110.

56. Schulz R, Beach SR, Ives DG, Martire LM, Ariyo AA, Kop WJ. Association between depression and mortality in older adults: the Cardiovascular Health Study. Arch Intern Med. 2000;160(12):1761-1768.

57. Barefoot JC, Schroll M. Symptoms of depression, acute myocardial infarction, and total mortality in a community sample. Circulation. 1996;93(11):1976-1980.

58. St John PD, Montgomery PR. Do depressive symptoms predict mortality in older people? Aging Ment Health. 2009;13(5):674-681.

59. Penninx BW, Geerlings SW, Deeg DJ, van Eijk JT, van Tilburg W, Beekman AT. Minor and major depression and the risk of death in older persons. Arch Gen Psychiatry. 1999;56(10):889-895.

60. Yasuda N, Mino Y, Koda S, Ohara H. The differential influence of distinct clusters of psychiatric symptoms, as assessed by the general health questionnaire, on cause of death in older persons living in a rural community of Japan. J Am Geriatr Soc. 2002;50(2):313-320.

61. Hölttä EH, Laakkonen ML, Laurila JV, Strandberg TE, Tilvis RS, Pitkala KH. Apathy: prevalence, associated factors, and prognostic value among frail, older inpatients. J Am Med Dir Assoc. 2012;13(6): 541-545.

62. Vilalta-Franch J, Calvo-Perxas L, Garre-Olmo J, Turro-Garriga O, Lopez-Pousa S. Apathy syndrome in Alzheimer's disease epidemiology: prevalence, incidence, persistence, and risk and mortality factors. J Alzheimers Dis. 2013;33(2):535-543.

63. Roest AM, Thombs BD, Grace SL, Stewart DE, Abbey SE, de Jonge P. Somatic/affective symptoms, but not cognitive/affective symptoms, of depression after acute coronary syndrome are associated with 12-month all-cause mortality. J Affect Disord. 2011;131(1-3):158-163.

64. Schiffer AA, Pelle AJ, Smith OR, Widdershoven JW, Hendriks EH, Pedersen SS. Somatic versus cognitive symptoms of depression as predictors of all-cause mortality and health status in chronic heart failure. J Clin Psychiatry. 2009;70(12):1667-1673.

65. Ariyo AA, Haan M, Tangen CM, et al. Depressive symptoms and risks of coronary heart disease and mortality in elderly Americans. Circulation. 2000;102(15):1773-1779.

66. Strine TW, Mokdad AH, Dube SR, et al. The association of depression and anxiety with obesity and unhealthy behaviors among communitydwelling US adults. Gen Hosp Psychiatry. 2008;30(2):127-137.

67. Eurelings LS, Jaccard J, van Charante EP, et al. The mediating role of cardiovascular risk factors in the relationship between symptoms of apathy and incident cardiovascular disease in community-dwelling older individuals. Int Psychogeriatr. 2016;28(4):669-679.
68. Meng L, Chen D, Yang Y, Zheng Y, Hui R. Depression increases the risk of hypertension incidence: a meta-analysis of prospective cohort studies. J Hypertens. 2012;30(5):842-851.

69. Mezuk B, Eaton WW, Albrecht S, Golden SH. Depression and type 2 diabetes over the lifespan: a meta-analysis. Diabetes Care. 2008;31(12):2383-2390.

70. Joynt KE, Whellan DJ, O'Connor CM. Depression and cardiovascular disease: mechanisms of interaction. Biol Psychiatry. 2003;54(3):248-261.

71. Tully PJ, Debette S, Mazoyer B, Tzourio C. White matter lesions are associated with specific depressive symptom trajectories among incident depression and dementia populations: Three-City Dijon MRI Study. Am J Geriatr Psychiatry. 2017;25(12):1311-1321.

72. Allan CL, Sexton CE, Filippini N, et al. Sub-threshold depressive symptoms and brain structure: a magnetic resonance imaging study within the Whitehall II cohort. J Affect Disord. 2016;204: 219-225.

73. Yao H, Takashima Y, Araki Y, Uchino A, Yuzuriha T, Hashimoto M. Leisure-time physical inactivity associated with vascular depression or apathy in community-dwelling elderly subjects: the Sefuri study. J Stroke Cerebrovasc Dis. 2015;24(11):2625-2631.

74. Wang L, Leonards CO, Sterzer P, Ebinger M. White matter lesions and depression: a systematic review and meta-analysis. JPsychiatr Res. 2014;56: $56-64$.

75. Nebes RD, Vora IJ, Meltzer CC, et al. Relationship of deep white matter hyperintensities and apolipoprotein E genotype to depressive symptoms in older adults without clinical depression. Am J Psychiatry. 2001;158(6):878-884.

76. World Health Organization. The top 10 causes of death. 2017. Available from: http://www.who.int/mediacentre/factsheets/fs310/en. Accessed February 4, 2018.

77. Eurelings LS, Richard E, Eikelenboom P, van Gool WA, van Charante EP. Low-grade inflammation differentiates between symptoms of apathy and depression in community-dwelling older individuals. Int Psychogeriatr. 2015;27(4):639-647.

78. Fones CS. Distinguishing apathy syndromes from vascular depression. Arch Gen Psychiatry. 1998;55(9):844-845.

79. Marin RS, Firinciogullari S, Biedrzycki RC. Group differences in the relationship between apathy and depression. J Nerv Ment Dis. 1994;182(4):235-239.

80. Hollocks MJ, Lawrence AJ, Brookes RL, et al. Differential relationships between apathy and depression with white matter microstructural changes and functional outcomes. Brain. 2015;138(Pt 12): 3803-3815.

81. Lanctot KL, Aguera-Ortiz L, Brodaty H, et al. Apathy associated with neurocognitive disorders: recent progress and future directions. Alzheimers Dement. 2017;13(1):84-100.

82. Vlasnik JJ, Aliotta SL, DeLor B. Medication adherence: factors influencing compliance with prescribed medication plans. Case Manager. 2005;16(2):47-51.

83. Barnhart WJ, Makela EH, Latocha MJ. SSRI-induced apathy syndrome: a clinical review. J Psychiatr Pract. 2004;10(3):196-199.

84. Berman K, Brodaty H, Withall A, Seeher K. Pharmacologic treatment of apathy in dementia. Am J Geriatr Psychiatry. 2012;20(2):104-122.

85. Harrison F, Aerts L, Brodaty H. Apathy in dementia: systematic review of recent evidence on pharmacological treatments. Curr Psychiatry Rep. 2016;18(11):103.

86. Coupland C, Dhiman P, Morriss R, Arthur A, Barton G, Hippisley-Cox J. Antidepressant use and risk of adverse outcomes in older people: population based cohort study. BMJ. 2011;343:d4551.

87. Kok RM, Reynolds CF 3rd. Management of depression in older adults: a review. JAMA. 2017;317(20):2114-2122. 
Clinical Epidemiology is an international, peer-reviewed, open access, online journal focusing on disease and drug epidemiology, identification of risk factors and screening procedures to develop optimal preventative initiatives and programs. Specific topics include: diagnosis, prognosis, treatment, screening, prevention, risk factor modification,

Submit your manuscript here: https://www.dovepress.com/clinical-epidemiology-journal
Dovepress

systematic reviews, risk and safety of medical interventions, epidemiology and biostatistical methods, and evaluation of guidelines, translational medicine, health policies and economic evaluations. The manuscript management system is completely online and includes a very quick and fair peer-review system, which is all easy to use. 